\title{
Investigating the Neural Correlates of Percepts Using Magnetoencephalography and Magnetic Source Imaging
}

\author{
Thomas Hartmann, Nathan Weisz, Winfried Schlee, and Thomas Elbert(®)
}

\begin{abstract}
Magnetoencephalography (MEG) has become an important tool for neuroscientists. The high temporal resolution and the low signal-to-noise ratio of MEG provide advantages that other neuroscientific methods do not. Owing to recent findings concerning the relationship between perception and neuronal oscillations, more attention is being drawn to the importance of MEG. This chapter provides an introduction to oscillatory brain dynamics and outlines the fundamental and recent research on this topic. It also includes an overview of the basic principles of MEG and compares MEG with other neuroscientific methods such as imaging techniques like functional magnetic resonance imaging, positron emission tomography and electroencephalography. Finally, as an example of the application of MEG in current research, a short review of our work on tinnitus is provided, including links to current research on general perception.
\end{abstract}

\section{Perception in Relation to Oscillatory Brain Dynamics}

Imagine for a moment a barking dog. It is easy to conjure up an image of the dog in our mind and to vividly add sensory and emotional content to this image. The image is a percept reconstructed from memory and it may be so vivid that we might perceive changes in peripheral physiological response such as sweating or a racing heart beat. Elements of the underlying complex network of this cognitive process have been formed by our own unique experiences in life and are modified each time we retrieve the information and each time we re-experience similar situations.

Multiple complex components of the brain are involved in this process and various pieces of sensory information related to this memory are utilized. For instance, the barking dog may be perceived acoustically at first, i.e., processed by the auditory

\section{T. Elbert}

Department of Psychology, University of Konstanz, Universitätsstraße 10, 78464 Konstanz, Germany

thomas.elbert@uni-konstanz.de 
system, which decodes sounds by segmenting and integrating temporal bits from the incoming stream of sound. Depending on the memory or image being recalled, noises such as the zoom of cars passing by, which are unrelated to the focus of attention (in this case the barking dog), might be suppressed. On another level, the visual level, we might try to determine the location of the dog and to retrieve additional information by trying to visualize the dog and its whereabouts. The brain might then perform cross-modal connections between the visual percept of the dog and the auditory percept of the dog's barking to create a whole picture, or Gestalt. But how does this happen? How does the brain code and process incoming information? How does it recognize a certain object like a dog? How does the Gestalt of a dog come into our awareness?

One of the key processes related to mentally conjuring an object is one's attention devoted to the corresponding sensory input that evokes the neural pattern underlying the Gestalt. This requires that attention be devoted to only one object at a time. However, one's attention may switch quickly between different objects or ongoing input processes and an individual may be aware of two different objects simultaneously, such as the dog and the car passing by. In this case, how can we avoid mixing the firing patterns of one certain type of movement (columns in V5) or a color (V4) of one object with those of another object?

In other words, one of the fundamental problems in the neurophysiology of cortical sensory coding is understanding how local cortical activity, which occurs in clusters of neurons with similar properties (columns, barrels, areas), leads to a unique and globally coherent percept of objects. In other words, how does the brain process large amounts of information simultaneously and in such a way that individual objects are recognized and transformed into a recognizable "Gestalt"? This problem seems particularly intriguing as there is no single area in the cortex where all processing pathways converge. The problem of how a subset of sensory information is selected to form the representation of a given object, the so-called binding problem, is complicated by the fact that for normal visual processing multiple objects must be represented simultaneously and in a hierarchical structure. Thus, any mechanism designed to solve the binding problem must be able to selectively "tag" feature-selective neurons that code for one particular object and, additionally, to demarcate the responses to one object from any simultaneous responses to other objects in order to avoid the illusory conjunction of features (von der Malsburg 1981).

A solution to the binding problem has been proposed by a number of researchers (Abeles 1982; von der Malsburg and Schneider 1986; von der Malsburg 1981) who suggest that neurons responding to the same object might synchronize their discharges with a temporal precision of a few milliseconds. In contrast, no synchronization should occur between cells encoding features of different objects. This concept complements and extends the classical notion of object representation by distributed neuronal assemblies (Hebb 1949). As in the Hebb model, representations are generated in a highly flexible and economic manner because any neuron can, at different times, participate in a number of different assemblies. Thus, new objects can readily be encoded by new patterns of activity in the same set of neurons. In principle, each of these neurons individually needs to encode primitive object features only. As the 
temporal aspects of activity become available as an additional coding dimension, the binding mechanism combines these advantages with the possibility of coactivating multiple object representations.

Experimental studies with humans and animals provide support for this concept of binding by synchronization. Several studies have demonstrated that spatially separate cells within the primary visual area can synchronize their spike discharges in both anaesthetized and awake cats (Eckhorn et al. 1988; Engel et al. 1990; Gray and Singer 1989; Gray et al. 1989; Michalski et al. 1983; Ts'o et al. 1986; summarized in Pantev et al. 1994). In most cases, the recorded cells synchronize with zero phase lag, which holds even if the recording sites are separated by more than $7 \mathrm{~mm}$ (Engel et al. 1990; Gray and Singer 1989). It was found that synchronization over these larger distances within the visual cortex of the cat only occurs when the respective neurons engage in oscillatory firing with a frequency of approximately $40 \mathrm{~Hz}$, and not when neurons fire more irregularly. Therefore, it has been suggested that gamma oscillations may function as a carrier for long-range synchronization (Engel et al. 1992). An important finding is that both within and across sensory areas response synchronization depends critically on the stimulus configuration. It was recently demonstrated that spatially separate cells in the visual cortex of the cat show strong synchronization only if they respond to the same visual stimulus. However, if responding to two independent stimuli, the cells fire in a less correlated manner or even without any fixed temporal relationship (Engel et al. 1991a, b; Gray and Singer 1989). Correlated firing has also been reported to occur between neurons of the primary and the secondary visual area (Eckhorn et al. 1993; Munk et al. 1993). Importantly, the stimulus dependence of neuronal interactions was confirmed in awake monkeys (Kreiter and Singer 1992). Testing this hypothesis in humans, Melloni et al. (2007) found increases in long-range gamma synchronization when subjects were presented with visible words, compared with a condition of subliminal word processing. An important extension to this concept has been suggested recently (Supp et al. 2007). The authors investigated the directionality of widespread cortical networks while familiar and unfamiliar pictures were viewed. Unfamiliar pictures entailed only a small number of unilateral connections. In contrast, familiar pictures entailed a widespread network of reciprocal (feed-forward and feed-backward) connections. These observations support the hypothesis that correlated firing between remote brain areas could provide a dynamic binding mechanism which permits the formation of assemblies in a flexible manner.

When we return to the seemingly "simple" example of a barking dog, we realize that multiple pieces of information need to be simultaneously analyzed by many specialized subsystems. Even though neuronal synchrony as a crucial mechanism in the formation of object-related cell assemblies was theoretically recognized by the early 1980s, it long awaited empirical validation. Recording from area 17, Gray and Singer (1989) observed synchronous firing of groups of neurons within a cortical column when presented with an optimal stimulus (slowly moving bars). The rate of simultaneous discharge lasting a few hundred milliseconds was periodic at approximately $30-60 \mathrm{~Hz}$, which falls into the so-called gamma frequency range (the boundaries of which are vaguely defined but usually fall within $30-100 \mathrm{~Hz}$ ). 


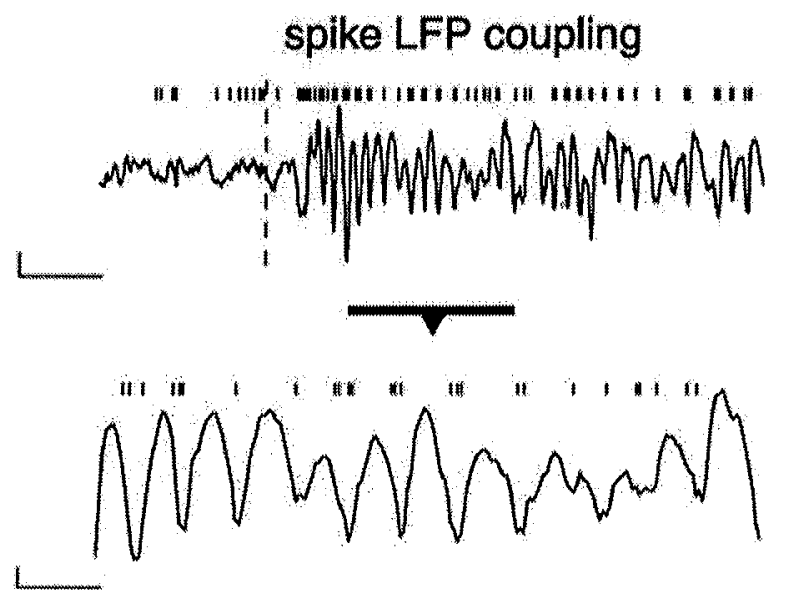

Fig. 1 Top: The multiunit spike recordings and the local field potentials $(L F P)$ at the same recording sites. The vertical dashed line shows the stimulus onset. Bottom: Expanded plot of the $200 \mathrm{~ms}$ marked in the top plot showing phase locking between multiunit spike recordings and the LFP. (Adapted from Siegel and König 2003.)

Importantly, local field potentials (LFPs) recorded with the same electrodes show an oscillatory modulation at the same rate, where a distinct phase of an oscillatory cycle coincides with the discharges (Fig. 1). This was later confirmed in several other studies (Fries et al. 2001; Gruber et al. 1999; Keil et al. 1999; Müller et al. 1996; Pulvermüller et al. 1999; Siegel and König 2003) and is of great importance in understanding the multitude of perception-related gamma-band results reported in magnetoencephalography (MEG) and electroencephalography (EEG) literature (Keil et al. 2001). If such flexible synchrony between cell assemblies actually existed in the brain it might be possible to track it using MEG-based technology, as outlined in more detail later in this chapter.

In their 1995 review, Singer and Gray (1995) differentiated five spatial scales at which synchronization putatively occurs:

(1) Same cortical column

(2) Different cortical columns

(3) Different cortical areas

(4) Two hemispheres

(5) Different sensory and motor modalities

With the use of EEG and MEG, macroscopic forms of synchronization can be studied, corresponding approximately to scales $2-5$ from the above list. Regarding scale 2 , of course a sufficient number of cortical columns must be involved because more than 10,000 neurons have to synchronize their activity so that the LFP is strong enough to generate an externally recordable signal. Scale 2 would be what EEG/MEG researchers call "local" synchrony (even though from an invasive viewpoint this would already be quite distant), with the single-column level being inaccessible by their methods. From the list it can be furthermore taken that 
synchrony exceeds the "local" level to include so-called long-range connections (scales 3-5). Indeed such long-range synchronies, putatively mediating integration of information from different brain regions, have been experimentally shown to exist, measured by intracranial and also noninvasive approaches such as EEG and MEG (Melloni et al. 2007; reviewed in Varela et al. 2001). Just recently, it was suggested that synchronization between neuronal cell assemblies not only binds the activity of neuronal cell assemblies but also leads to spike-time-dependent plasticity in the cortex and thus governs long-term effects on cognitive functions (Womelsdorf et al. 2007).

This being said, in order to gain a relatively "complete" picture of electrophysiological processes, the strengths from intracranial EEG and noninvasive EEG/MEG must be combined. Whereas intracranial recordings can score out the details regarding spatial accuracy and also the relation between oscillatory activity and discharges, MEG and EEG provide the "big picture." In this chapter we will concentrate on the MEG method. We will compare it with other established neuropsychological procedures like functional magnetic resonance imaging (fMRI) and positron emission tomography (PET) to show the fundamental differences between them. Finally, we will exemplify the utility of MEG in investigating oscillatory brain activity with an example from our own research.

\section{Magnetoencephalography}

\subsection{Basics of MEG}

The excited portion of the dendritic tree represents a local source of current. The intracellular current flow produces a magnetic field that can be measured as magnetoencephalographic signals. At different locations the current penetrates through the cell membrane such that the circuit can be closed over the volume conductor i.e., by current pathways through extracellular body tissue. The bioelectric potentials that originate from the volume currents are recorded as an electroencephalogram, which refers to the voltage derived from two electrodes attached to the surface of the scalp. In a homogenous volume conductor, the magnetic fields produced by volume currents sum up to zero. As body tissue is not homogenous, there may be some contribution from volume currents, referred to as secondary sources. Usually such contributions are, however, small and can be neglected (Elbert 1998).

The magnetic fields produced by intracellular currents flowing in neighboring dendritic trees of pyramidal neurons towards the soma mostly have the same orientation and therefore the sum is of a measurable size. If enough neighboring cells show this synchronized behavior (about 20,000-50,000), the emitted field is strong enough to be measured by MEG.

To measure the biomagnetic fields, sensors are needed to detect fields as small as femtotesla. MEG uses so-called superconducting quantum interference device (SQUIDs) to detect the current that is induced by the magnetic field and convert it 
to voltage. The SQUIDs are located inside a larger apparatus that encompasses the skull during measurement. This helmet-like apparatus is constantly cooled by liquid helium to maintain the superconductor effect. To decrease the effects of urban magnetic activity, such as from traffic or other large magnetic fields, the entire MEG instrument is installed inside a magnetically shielded room. Furthermore, additional sensors are installed within the outer layer of the helmet in order to measure remaining noise that can later be subtracted from the measurements of the internal sensors which are measuring close to the skull of the subject.

One serious limitation of MEG and EEG is that the localization of the neural generators cannot be directly and unequivocally derived from the measured signal. Because the recorded data is a $2 \mathrm{D}$ projection of activity that has three spatial dimensions, source activity has to be properly modeled. Source modeling faces similar issues as the visual system that has to derive coherent objects and their location in three dimensions is based on a $2 \mathrm{D}$ retinotopic representation. Generally there is no unique solution to this problem, as proven by Helmholtz. But the widely held assumption that the lack of a reliable method of source localization prevents one from making confident inferences about the underlying neural generators cannot be supported. In the cases of both MEG and EEG, additional information, e.g., derived from neuroanatomical knowledge, can be used to constrain the solution space, making it possible to deduce the best possible solution to the problem.

\subsection{Advantages of MEG over Imaging Methods}

From the initial enthusiasm that resulted when modern neuroimaging techniques like fMRI and PET were developed, one would have though that these techniques would soon replace EEG/MEG or that electrophysiological methods would have to be combined with neuroimaging methods. This was mainly owing to the spatial resolution provided by neuroimaging methods, especially in deep regions of the brain. However, in recent years EEG and MEG have witnessed a true renaissance stemming from three developments: (1) the inherent limits regarding the temporal resolution of neuroimaging methods are more than $1 \mathrm{~s}$, whereas electrophysiological methods reflect neuronal activity in real time (millisecond range); (2) the widespread availability of high-density EEG/MEG systems, which combined with advances in localization techniques (see later) have improved the spatial resolution of noninvasive electrophysiological methods; (3) even though there appear to be some correlations between signals recorded by fMRI and LFPs (Logothetis 2007), it is far from clear what aspects related to neuronal activity may actually modulate hemodynamic changes (Burke and Bührle 2006). In contrast to MEG, PET and single photon emission computed tomography both require the use of radioactive substances. Compared with fMRI, MEG has the advantage that no magnetic field is emitted by the machine; therefore, there is no risk of harm to the subjects or staff from metal parts that are brought close to the machine. 
Instead of being offered up as an alternative to one another, or dismissing the usability of a specific technique such as MEG or EEG, the various neuroimaging methods should not be seen as mutually exclusive; rather, they should be seen as complementary. For example, fMRI and PET are of great value in clinical settings and have been shown to be very useful with patients who have suffered strokes. In these cases, fMRI and PET can be used to visualize nonfunctional areas of the brain and to identify cortical areas that can be rehabilitated through specific intervention.

\subsection{Comparison of MEG and EEG}

While theory suggests that similar physiological processes underlie EEG and MEG, the signals generally provide different information when used in real measurements and therefore are ideally combined to extract maximal information. This may be counterintuitive since the neuromagnetic or MEG signals can be similar in appearance to those of EEG. The EEG electrodes cover only a relatively small area compared with the distance between two electrodes and, thus, EEG is vulnerable to spatial aliasing in which physiologically distinct signals become indistinguishable in the measurement. For shallow sources that have the highest spatial frequencies, 100 or more electrodes are needed (when equally spaced across the head's surface) in order to avoid spatial aliasing (Junghöfer et al. 1997). When comparing highresolution (approximately 128 electrodes) EEG and MEG, the following differences can be noted:

- Improper fixation or location of EEG electrodes produces artifacts or errors in the source estimation (e.g., distortion of the interpolated surface potential and consequently erroneous "ghost sources").

- Artifacts are more common in EEG than in MEG as EEG may be affected by movement of electrodes, electrode drift and volume-conducted electrocardiography. Similarly, ocular artifacts are also more remarkable for EEG than for MEG.

But the greatest difference stems from the selectivity of MEG with respect to the orientation of sources. As mentioned, the electroencephalogram results from the extracellular volume currents triggered mainly by postsynaptic potentials. The magnetoencephalogram, in contrast, arises from the intracellular branch of this process, i.e., from the currents that flow within the dendrite to the soma. Thereby, MEG is mainly sensitive to currents flowing tangentially to the surface of the scalp and to a lesser degree - about 10\% - to radial sources. As a consequence EEG and MEG are affected differently by averaging: If sources vary across trials and appear in different cerebral regions from trial to trial their impact on the event-related brain responses will be suppressed by averaging. Thereby, this "biological noise" is more strongly reduced for tangential sources than for radial ones (as tangential sources in opposing walls of a sulcus may partially cancel each other out, leaving only the radially directed currents in the average). Sources in the primary and secondary sensory projection areas, such as Brodmann areas $3 \mathrm{~b}$ (somatosensory), 41/42 (auditory) or 
17 (visual), are primarily tangentially oriented and are consistently evoked in each trial. Consequently, for such sources activated early in the information processing, the signal-to-noise ratio is considerably higher for MEG measurements than for EEG measurements.

A high-resolution EEG system costs about $€ 200,000$. An MEG system, which starts at about $€ 1,000,000$, is not only considerably more expensive to purchase, but also requires around $10 \mathrm{l}$ of liquid helium per day for operation, which totals about another $€ 30,000$ per year. The preparation for EEG, on the other hand, is more labor-intensive and, owing to a greater sensitivity to artifacts, requires larger sample sizes.

Another advantage of MEG is the fact that the magnetic fields penetrate tissue mostly undistorted, whereas the volume current of EEG must penetrate the cerebrospinal fluid, the meninges, the skull and the skin in a correspondingly complicated spatial pattern. Moreover, in order to realize low impedances between the skin and the electrodes, conductive agents such as gels or creams are needed. These agents might lead to a blurring of signals if two or more adjacent electrodes connect to each other through the conductive agent. This is particularly true with EEG systems that utilize a large number of electrodes.

The magnetic field measured by MEG passes through the outer layers of the head almost unaffected. This is also an advantage for source modeling as underlying models can be much simpler than with EEG (indeed EEG source solutions can be very sensitive to head-model misspecifications (Plis et al. 2007). In addition, there is no need for conductive agents that might blur the spatial information.

Furthermore, as we have already explained, MEG and EEG measure different properties. While EEG measures the volume conduction, meaning the current that counterbalances the internal current flow of many different neurons, MEG is able to directly measure the intracellular currents. As volume conduction spreads out across the surface, and is influenced by other electric signals that may lie at some distance, the currents can be greatly disturbed. The intracellular current is immune to these influences and does not blur at the surface and/or in the brain, leading to a higher spatial accuracy.

Another advantage further increasing the signal-to-noise ratio stems from (1) the columnar organization and folded layout of the cortex and (2) the fact that MEG is almost blind to radial sources. Two thirds of the cortex lie in sulci, which means that the chance of the desired signal being oriented tangentially is higher than that of the desired signal being exclusively radially oriented. This can be regarded as a kind of spatial filter, leading to more focal effects. In contrast to EEG, which records this signal, MEG is blind to this part of the signal, thus enhancing the signal-to-noise ratio. The remaining sources of noise are mostly tangentially oriented and thus lie in the walls of the sulci. As each potential noise source has another noise source at the opposite wall of the sulci, these two most likely cancel each other out. These enhancements in signal quality make it possible to detect signals with MEG that would not be detectable or that would be difficult to separate from other parallel processes using EEG. 


\section{Unraveling the Neuronal Correlates of Tinnitus with MEG}

In this section we present, as an example, a series of studies in which EEG and MEG were used to investigate abnormalities of the cortical activity in chronic tinnitus subjects. Since subjective tinnitus is described as a conscious perception of a sound in the absence of a physical sound source, research on this topic has general implications regarding neuronal activity underlying percepts. The perceived sound is typically described by the patients as a tone, a hissing, a roaring noise or some combination of these sounds. Transient tinnitus is quite common in the general population and typically lasts a few seconds to a few hours or even a few days. However, a diagnosis of chronic tinnitus is made when the tinnitus lasts uninterrupted for more than 3 months. About 5-15\% of the population in Western societies (Heller 2003) report chronic tinnitus and in $1-3 \%$ the tinnitus affects the quality of life. This can occur in the form of sleep disturbances, impaired ability to concentrate at work, difficulties in social interactions and psychiatric distress (Dobie 2003).

As tinnitus is a percept without a physical stimulus, the question arises whether it is possible to find changes in the neuronal oscillations which might reflect synchronized cortical activity as described at the beginning of the chapter. Of the five spatial scales of synchronization mentioned previously in this chapter, scales 2-5 are most relevant for MEG-based research and for which functional neuroimaging techniques are agnostic because of their low temporal resolution.

We believe that a deafferentation of auditory brain regions is necessary in order for tinnitus to develop (Eggermont and Roberts 2004; Saunders 2007; Weisz et al. 2006). However, we also know that deafferentation is not a sufficient condition for the development of tinnitus. This is owing to the fact that not all patients with a profound hearing loss (and thus deafferantation of the auditory system) also suffer from tinnitus (König et al. 2006). Furthermore, treatment approaches aiming at altering the changed tonotopy have proven to be efficient in reducing the perception of tinnitus (Dohrmann et al. 2007a, b; Flor et al. 2004).

As with the barking dog, the conscious perception of the tinnitus sound is most likely associated with a long-range interaction of remote brain areas. We think that the critical condition for evoking such a widespread network is a reduction of cortical inhibition (Weisz et al. 2007a). Such a loss of inhibition would lead to spontaneously synchronized brain activity within the auditory cortex as well as between brain regions relevant for the processing of attentional and emotional aspects of tinnitus. When we speak of neuronal oscillations we must be more specific and will classify them according to the scale outlined at the beginning of this chapter (Singer and Gray 1995).

We first examined whether there was altered local synchrony (scale 2 on the Singer and Gray scale) by acquiring a resting-state MEG measurement where subjects were asked to lie still with their eyes open. The data were then analyzed in the frequency domain and revealed an enhancement in lower frequencies (below $4 \mathrm{~Hz}$ ) accompanied by a decrease of energy in the alpha band $(8-12 \mathrm{~Hz}$ ) (Weisz et al. 2005). Both changes were located in temporal regions that are relevant for auditory processing. The extent of these changes showed a high correlation with decrease 
in tinnitus distress. The functional importance of the frequency bands is supported by an EEG neurofeedback study showing a strong relationship between the extent patients were able to normalize their spontaneous activity spectra and the reduction of tinnitus intensity. In this study there were even two cases in which the tinnitus sensation completely disappeared (Dohrmann et al. 2007b).

In Sect. 1, we mentioned that perception is accompanied by local and global synchronization of firing expressed by the gamma-band rhythm of the LFP (more than $30 \mathrm{~Hz}$ ). It thus seems logical to investigate whether tinnitus, being a phantom percept, leads to changes in the gamma oscillations (Weisz et al. 2007b). Our study examining this effect yielded three important results:

(1) In subjects with tinnitus, the overall gamma-band activity was increased.

(2) Individuals with unilateral tinnitus showed more gamma-band activity on the contralateral side to the tinnitus than on the ipsilateral side.

(3) Particularly in control subjects we found a strong correlation in the time course between slow-wave and gamma activity.

These results are of great relevance for the understanding of tinnitus as we were able to show alterations of local synchronization and thus an objective measurement for an otherwise subjective percept. Generally these findings underline the importance of gamma-band activity in the generation of conscious percepts.

We then moved to a more global approach to assess the connectivity between different cortical areas in tinnitus subjects. Schlee et al. (2007) stimulated tinnitus and control subjects with $40-\mathrm{Hz}$ amplitude-modulated tones. The highest one was close to the tinnitus frequency, while the other two were 1.1 and 2.2 octaves lower. While every stimulus evokes a transient reaction that lasts for about $500 \mathrm{~ms}$ in EEG and $\mathrm{MEG}$, amplitude-modulated or frequency-modulated stimuli evoke responses at the modulation frequency that last until stimulus offset. While other studies have only dealt with the amplitude and thus local synchronization, Schlee et al. (2007) examined whether changes in the global synchronization could be found. For this purpose they applied a source montage on MEG data consisting of eight fixed regional sources each at a prominent location of the cortex (anterior cingulate cortex, posterior cingulate cortex, left and right frontal, temporal and parietal) and calculated the phase coherence between these sources for the $40-\mathrm{Hz}$ response.

Phase coherence is a measurement to assess whether two sources are synchronously activated in each trial. It is calculated by subtracting the phases of source A from the phases of source B for each trial. If the variance of these differences is low, it can be assumed that these two sources are synchronously active and form a functionally related network. The phase coherence of each individual was then correlated with the individual's tinnitus distress as assessed with the German version of the Tinnitus Questionnaire (Göbel und Hiller). This revealed a strong positive correlation between tinnitus intrusiveness and phase coherence between the right parietal and anterior cingulate regions (Fig. 2). The inverse was found for the correlation between tinnitus intrusiveness and the connection of right frontal and anterior cingulate regions. It is important to note that these effects were only present for the stimulus whose frequency was close to the tinnitus tone reported by the subjects. 

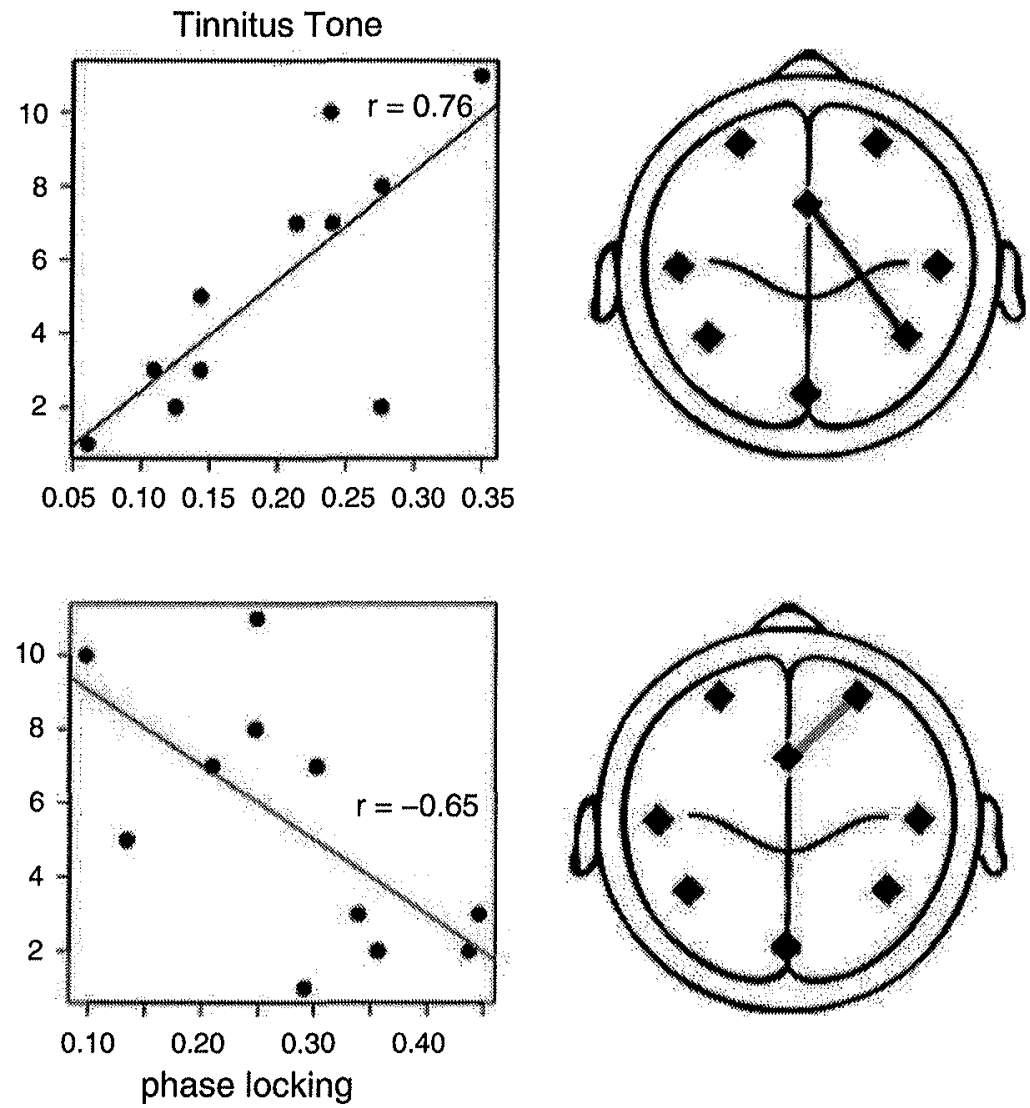

Fig. 2 Interregional connectivities with an association between tinnitus intrusiveness and phase synchronization. The top plot shows the scatterplot of the interregional connectivity between the right parietal and the anterior cingulate cortex across all stimulation conditions. Subjective ratings were positively correlated with the interregional phase synchronization when stimulated with the tinnitus tone. The bottom plot depicts the connectivity between right frontal and anterior cingulate cortex. The correlation between tinnitus intrusiveness and phase synchrony was negative. (Adapted from Schlee et al. unpublished results.)

Interestingly, these findings are in accordance with new results made with an advanced magnetic resonance tomography based imaging technique called diffusion tensor imaging. Lee et al. (2007) were able to show differences in the connections between temporal and frontal regions between tinnitus and control subjects. They also linked their findings to other functional imaging studies that were able to show contributions of emotional and memory systems, both being located in frontal regions. These findings also agree with those reported here.

Although these findings are of great importance for understanding tinnitus, it is also possible to link these findings to research regarding general perception. In a recent article by our research workgroup, a model integrating current knowledge 
about oscillatory brain activity (Miller 2007) and abnormalities in tinnitus patients was proposed (Weisz et al. 2007a). The effect that perception of any kind leads to a decrease of energy in the alpha band and an increase in the gamma band is in accordance with findings in tinnitus patients who seem to show a chronic increase in these frequency bands.

Although we are still far from a deep understanding of the mechanisms behind tinnitus, we have shown here that MEG has provided insights that would not have otherwise been impossible to attain. These insights will give rise to new therapeutic approaches that might ultimately lead to effective treatment modalities.

\section{Summary}

Neuronal oscillations have become a valuable and important tool for neuroscientists to research neuronal activity which is too fast to be measured using methods that depend on blood flow or blood oxygenation. Tinnitus research is one of the best examples of the usefulness of MEG as there is great potential for new and important insights that will increase our future understanding of the condition and which may result in viable treatment options. MEG, although more expensive than EEG, provides scientists with more accurate measurements - in the time and the spatial domain - and an enhanced signal-to-noise ratio, thus providing even greater insights into our understanding and knowledge of these topics.

\section{References}

Abeles M (1982) Role of the cortical neuron: integrator or coincidence detector? Isr J Med Sci 18:83-92

Burke M, Bührle C (2006) BOLD response during uncoupling of neuronal activity and CBF. Neuroimage 32:1-8

Dobie RA (2003) Depression and tinnitus. Otolaryngol Clin North Am 36:383-388

Dohrmann K, Elbert T, Schlee W, Weisz N (2007a) Tuning the tinnitus percept by modification of synchronous brain activity. Restor Neurol Neurosci 25:371-378

Dohrmann K, Weisz N, Schlee W, Hartmann T, Elbert T (2007b) Neurofeedback for treating tinnitus. Prog Brain Res 166:473-485

Eckhorn R, Bauer R, Jordan W, Brosch M, Kruse W, Munk M, Reitboeck HJ (1988) Coherent oscillations: a mechanism of feature linking in the visual cortex? Multiple electrode and correlation analyses in the cat. Biol Cybern 60:121-130

Eckhorn R, Frien A, Bauer R, Woelbern T, Kehr H (1993) High frequency (60-90 Hz) oscillations in primary visual cortex of awake monkey. Neuroreport 4:243-246

Eggermont JJ, Roberts LE (2004) The neuroscience of tinnitus. Trends Neurosci 27:676-682

Elbert T (1998) Neuromagnetism. In: Andrä W, Nowak H (eds) Magnetism in medicine. Wiley, New York, pp 190-262

Engel AK, König P, Gray CM, Singer W (1990) Stimulus-dependent neuronal oscillations in cat visual cortex: inter-columnar interaction as determined by cross-correlation analysis. Eur $\mathrm{J}$ Neurosci 2:588-606 
Engel AK, Kreiter AK, König P, Singer W (1991a) Synchronization of oscillatory neuronal responses between striate and extrastriate visual cortical areas of the cat. Proc Natl Acad Sci USA 88:6048-6052

Engel AK, König P, Singer W (1991b) Direct physiological evidence for scene segmentation by temporal coding. Proc Natl Acad Sci USA 88:9136-9140

Engel AK, König P, Kreiter AK, Schillen TB, Singer W (1992) Temporal coding in the visual cortex: new vistas on integration in the nervous system. Trends Neurosci 15:218-226

Flor H, Hoffmann D, Struve M, Diesch E (2004) Auditory discrimination training for the treatment of tinnitus. Appl Psychophysiol Biofeedback 29:113-120

Fries P, Reynolds JH, Rorie AE, Desimone R (2001) Modulation of oscillatory neuronal synchronization by selective visual attention. Science 291:1560-1563

Gray CM, Singer W (1989) Stimulus-specific neuronal oscillations in orientation columns of cat visual cortex. Proc Natl Acad Sci USA 86:1698-1702

Gray CM, König P, Engel AK, Singer W (1989) Oscillatory responses in cat visual cortex exhibit inter-columnar synchronization which reflects global stimulus properties. Nature 338:334-337

Gruber T, Müller MM, Keil A, Elbert T (1999) Selective visual-spatial attention alters induced gamma band responses in the human EEG. Clin Neurophysiol 110:2074-2085

Hebb DO (1949) The organization of behavior: a neuropsychological theory. Wiley, New York

Heller AJ (2003) Classification and epidemiology of tinnitus. Otolaryngol Clin North Am $36: 239-248$

Junghöfer M, Elbert T, Leiderer P, Berg P, Rockstroh B (1997) Mapping EEG-potentials on the surface of the brain: a strategy for uncovering cortical sources. Brain Topogr 9:203-217

Keil A, Müller MM, Ray WJ, Gruber T, Elbert T (1999) Human gamma band activity and perception of a gestalt. J Neurosci 19:7152-7161

Keil A, Gruber T, Müller MM (2001) Functional correlates of macroscopic high-frequency brain activity in the human visual system. Neurosci Biobehav Rev 25:527-534

Kreiter AK, Singer W (1992) Oscillatory neuronal responses in the visual cortex of the awake macaque monkey. Eur J Neurosci 4:369-375

König O, Schaette R, Kempter R, Gross M (2006) Course of hearing loss and occurrence of tinnitus. Hear Res 221:59-64

Lee Y, Bae S, Lee S, Lee J, Lee K, Kim M, Kim Y, Baik S, Woo S, Chang Y (2007) Evaluation of white matter structures in patients with tinnitus using diffusion tensor imaging. J Clin Neurosci 14:515-519

Logothetis NK (2007) The ins and outs of fMRI signals. Nat Neurosci 10:1230-1232

Melloni L, Molina C, Pena M, Torres D, Singer W, Rodriguez E (2007) Synchronization of neural activity across cortical areas correlates with conscious perception. J Neurosci 27:2858-2865

Michalski A, Kossut M, Turlejski K, Chmielowska J (1983) Responses of area 17 neurons in cats binocularly deprived by rearing in hoods. Acta Neurobiol Exp (Wars) 43:263-272

Miller R (2007) Theory of the normal waking EEG: from single neurones to waveforms in the alpha, beta and gamma frequency ranges. Int J Psychophysiol 64:18-23

Müller MM, Bosch J, Elbert T, Kreiter A, Sosa MV, Sosa PV, Rockstroh B (1996) Visually induced gamma-band responses in human electroencephalographic activity - a link to animal studies. Exp Brain Res 112:96-102

Munk MHJ, Nowak LG, Bullier J (1993) Spatio-temporal response properties and interactions of neurons in areas V1 and V2 of the monkey. Abstr Soc Neurosci 19:179.3

Pantev C, Eulitz C, Elbert T, Hoke M (1994) The auditory evoked sustained field: origin and frequency dependence. Electroencephalogr Clin Neurophysiol 90:82-90

Plis SM, George JS, Jun SC, Ranken DM, Volegov PL, Schmidt DM (2007) Probabilistic forward model for electroencephalography source analysis. Phys Med Biol 52:5309-5327

Pulvermüller F, Keil A, Elbert T (1999) High-frequency brain activity: perception or active memory? Trends Cogn Sci 3:250-252

Saunders JC (2007) The role of central nervous system plasticity in tinnitus. J Commun Disord $40: 313-334$ 
Schlee W, Weisz N, Dohrmann K, Hartmann T, Elbert T (2007) Unravelling the tinnitus distress network using single trial auditory steady-state responses. Int Congr Ser 1300:73-76

Siegel M, König P (2003) A functional gamma-band defined by stimulus-dependent synchronization in area 18 of awake behaving cats. J Neurosci 23:4251-4260

Singer W, Gray CM (1995) Visual feature integration and the temporal correlation hypothesis. Annu Rev Neurosci 18:555-586

Supp GG, Schlögl A, Trujillo-Barreto N, Müller MM, Gruber T (2007) Directed cortical information flow during human object recognition: analyzing induced EEG gamma-band responses in brain's source space. PLoS One 2:e684

Ts'o DY, Gilbert CD, Wiesel TN (1986) Relationships between horizontal interactions and functional architecture in cat striate cortex as revealed by cross-correlation analysis. $\mathrm{J}$ Neurosci 6:1160-1170

Varela F, Lachaux JP, Rodriguez E, Martinerie J (2001) The brainweb: phase synchronization and large-scale integration. Nat Rev Neurosci 2:229-239

von der Malsburg C (1981) The correlation theory of brain function. $\mathrm{p} 81$

von der Malsburg C, Schneider W (1986) A neural cocktail-party processor. Biol Cybern 54:29-40

Weisz N, Moratti S, Meinzer M, Dohrmann K, Elbert T (2005) Tinnitus perception and distress is related to abnormal spontaneous brain activity as measured by magnetoencephalography. PLoS Med 2(6):e153

Weisz N, Hartmann T, Dohrmann K, Schlee W, Norena A (2006) High-frequency tinnitus without hearing loss does not mean absence of deafferentation. Hear Res 222:108-114

Weisz N, Dohrmann K, Elbert T (2007a) The relevance of spontaneous activity for the coding of the tinnitus sensation. Prog Brain Res 166:61-70

Weisz N, Müller S, Schlee W, Dohrmann K, Hartmann T, Elbert T (2007b) The neural code of auditory phantom perception. J Neurosci 27:1479-1484

Womelsdorf T, Schoffelen J, Oostenveld R, Singer W, Desimone R, Engel AK, Fries P (2007) Modulation of neuronal interactions through neuronal synchronization. Science 316: $1609-1612$ 